\title{
Cocaine-but not methamphetamine-associated memory requires de novo protein synthesis
}

\author{
Yu-Min Kuo a , Keng Chen Liang ${ }^{\mathrm{b}}$, Hsiang-Hua Chen ${ }^{\mathrm{c}}$, Chianfang G. Cherng ${ }^{\mathrm{d}}$, \\ Hsueh-Te Lee ${ }^{e}$, Yinchiu Lin ${ }^{c}$, A-Min Huang ${ }^{f}$, Ruey-Ming Liao ${ }^{g}$, Lung Yu ${ }^{\text {c,e,f,* }}$ \\ ${ }^{a}$ Department of Anatomy and Cell Biology, National Cheng Kung University College of Medicine, Tainan 701, Taiwan, ROC \\ ${ }^{\mathrm{b}}$ Department of Psychology, National Taiwan University, Taipei 106, Taiwan, ROC \\ ${ }^{\mathrm{c}}$ Institute of Behavioral Medicine, National Cheng Kung University College of Medicine, Tainan 701, Taiwan, ROC \\ d Aviation Safety Training Center, Chinese Air Force Academy, Kangshan 820, Taiwan, ROC \\ ${ }^{\mathrm{e}}$ Institute of Basic Medical Sciences, National Cheng Kung University College of Medicine, Tainan 701, Taiwan, ROC \\ ${ }^{\mathrm{f}}$ Department of Physiology, National Cheng Kung University College of Medicine, Tainan 701, Taiwan, ROC \\ ${ }^{\mathrm{g}}$ Department of Psychology, National Chengchi University, Taipei 116, Taiwan, ROC
}

Received 25 April 2006; revised 29 June 2006; accepted 29 June 2006

Available online 14 August 2006

\begin{abstract}
Context-induced drug craving and continuous drug use manifest the critical roles of specific memory episodes associated with the drug use experiences. Drug-induced conditioned place preference (CPP) in C57BL/6J mouse model, in this regard, is an appropriate behavioral paradigm to study such drug use-associated memories. Requirement of protein synthesis in various forms of long-term memory formation and storage has been phylogenetically demonstrated. This study was undertaken to study the requirement of protein synthesis in the learning and memory aspect of the conditioned place preference induced by cocaine and methamphetamine, two abused drugs of choice in local area. Since pCREB has been documented as a candidate substrate for mediating the drug-induced neuroadaptation, the pCREB level in hippocampus, nucleus accumbens, and prefrontal cortex was examined for its potential participation in the formation of CPP caused by these psychostimulants. We found that cocaine $(2.5$ and $5.0 \mathrm{mg} / \mathrm{kg} / \mathrm{dose})$-induced CPP was abolished by the pretreatment of anisomycin $(50 \mathrm{mg} / \mathrm{kg} / \mathrm{dose})$, a protein synthesis inhibitor, whereas methamphetamine $(0.5 \mathrm{or} 1.0 \mathrm{mg} /$ $\mathrm{kg} / \mathrm{dose}$ )-induced CPP was not affected by the anisomycin pretreatment. Likewise, cocaine-induced CPP was mitigated by another protein synthesis inhibitor, cycloheximide $(15 \mathrm{mg} / \mathrm{kg} /$ injection) pretreatment, whereas methamphetamine-induced $\mathrm{CPP}$ remained intact by such pretreatment. Moreover, anisomycin treatment $2 \mathrm{~h}$ after each drug-place pairing disrupted the cocaine-induced CPP, whereas the same treatment did not affect methamphetamine-induced CPP. An increase of accumbal pCREB level was found to associate with the learning phase of cocaine, but not with the learning phase of methamphetamine. We further found that intraaccumbal CREB antisense oligodeoxynucleotide infusion diminished cocaine-induced CPP, whereas did not affect the methamphetamineinduced CPP. Taken together, these data suggest that protein synthesis and accumbal CREB phosphorylation are essential for the learning and consolidation of the cocaine-induced CPP, whereas methamphetamine-induced CPP may be unrelated to the synthesis of new proteins.
\end{abstract}

(C) 2006 Elsevier Inc. All rights reserved.

Keywords: Cocaine; Methamphetamine; pCREB; Conditioned place preference; Learning and memory; Accumbens

\footnotetext{
* Corresponding author. Present address: Behavioral Neuropharmacology Laboratory, Institute of Behavioral Medicine, National Cheng Kung University College of Medicine, Tainan, Taiwan 7001, ROC. Fax: + 88662095616.

E-mail address: lungyu@mail.ncku.edu.tw (L.Yu).
}

\section{Introduction}

A fundamental question residing in drug abuse research is how an organism switches from the sporadic and recreational use of drugs to continuous craving for drugs. These motivational changes resulted, in part, from 
the formation of memory associated with drug use (Cannon \& Bseikri, 2004). Most drug abuse studies, to date, adopt the motivational viewpoint and emphasize the euphoric/hedonic/reinforcing efficacy of drugs and associated changes. How learning and memory resulted from previous drug exposures affects subsequent drug use, however, remains scarcely explored. Understanding the formation mechanism of drug-associated memory may shed light on potential therapeutic approaches to effectively intervene with drug use-associated memory.

Cocaine, amphetamine and its analogs are primarily abused drugs of choice in North America and East Asia. In this study, we decided to examine the mechanism of the memory formation due to the psychostimulant use. Drug-induced conditioned place preference (CPP), i.e., the preference for a specific place cuing for drug availability over a neutral place, was used as a suggestive behavioral paradigm indicative of drug-associated memories. Previously, we and others have demonstrated that methamphetamine- and cocaine-induced place preferences were long-lasting and resistant to extinguish for weeks (Achat-Mendes, Anderson, \& Itzhak, 2003; Lien et al., 2004). Varieties of long-term memory were proved to be dependent on de novo protein synthesis (Ezzeddine \& Glanzman, 2003; Goelet, Castellucci, Schacher, \& Kandel, 1986; Kandel, 2001; Ramirez, Gandhi, Muzzio, \& Matzel, 1998; Stiedl, Palve, Radulovic, Birkenfeld, \& Spiess, 1999). However, other laboratories reported that certain long-term memories are unrelated to de novo protein synthesis (Flood \& Cherkin, 1987; Judge \& Quartermain, 1982; Kameyama, Nabeshima, \& Kozawa, 1986; Lattal \& Abel, 2001; Martinez, Jensen, \& McGaugh, 1981; Quevedo et al., 1999; Rainbow, Hoffman, \& Flexner, 1980). We therefore tested whether formation of methamphetamine- and cocaine-associated memory as seen in the CPP may require de novo protein synthesis.

Accrued evidence has posited the participation of mesolimbicortical dopaminergic systems in psychostimulantinduced addictive processes (Bonci, Bernardi, Grillner, \& Mercuri, 2003). The activation/phosphorylation of cyclic adenosine monophosphate response element binding protein (CREB), one of the transcription factors regulating the protein synthesis, is thought to be the critical, biological substrate mediating the psychostimulant-induced neuroadaptive modifications (Frank \& Greenberg, 1994; McClung \& Nestler, 2003). To examine the role of phosphorylated CREB (pCREB) in maintaining the psychostimulant-induced CPP, pCREB in hippocampus (a context learning-related region), nucleus accumbens (a primary dopaminergic target), and prefrontal cortex (also a dopaminergic target) were analyzed during and after the learning phase of psychostimulant-induced place preferences. Moreover, infusion of CREB antisense oligodeoxynucleotides into certain brain region was employed to examine the regional role of CREB in mediating the memory aspect of the druginduced CPP.

\section{Materials and methods}

\subsection{Animals}

Male C57BL/6J mice, 8 weeks of age (NCKUCM Lab Animal Center, Tainan, Taiwan), were housed four in one plastic cage with free access to food (Purina mouse chow, Richmond, IN) and tap water in a temperature $\left(22^{\circ} \mathrm{C}\right)$ - and humidity $(60 \%)$-controlled colony room and maintained on a $12 \mathrm{~h}$ light-dark cycle (lights on at 0700). This study was performed in accordance with the National Institutes of Health Guide for the Care and Use of Laboratory Animals. All procedures were approved by the local Animal Care Committee at the National Cheng Kung University College of Medicine.

\subsection{Drug-induced conditioned place preference}

Drug-induced CPP pairings and tests were conducted in a commercial unit designed for mouse (MedAssociates Inc., Georgia, VT). Each unit consisted of a center $(9 \times 13 \times 13 \mathrm{~cm})$ and two side compartments $(17 \times 13 \times 13 \mathrm{~cm})$. The side compartments were distinguished by three sets of cues: medium ( 160 Lux) vs. dim ( 40 Lux) roof light illuminations, black vs. white walls and ceilings, and steel bar grid vs. wire-mesh floors. The center was bright-lit with gray walls, ceiling and a gray platform floor. Automatic guillotine doors controlled passages between the center and the side compartments. Mouse location in each unit was monitored by photocell detectors aligned $1.5 \mathrm{~cm}$ above the floor across three compartments with $3 \mathrm{~cm}$ apart, connected via interface card to IBM-compatible PC and the time spent in each compartment was recorded and analyzed by MEDPC for Windows. Units were deodorized by a thorough cleaning with an isopropyl alcohol-rinsed paper towel wiping and followed by a dry process before each training and test. Drug-induced CPP training and test procedures were described in detail in our previous report (Lien et al., 2004). Briefly, mice received an intraperitoneal (i.p.) injection of methamphetamine hydrochloride, or cocaine hydrochloride and were immediately confined in one randomly assigned compartment of the units for $30 \mathrm{~min}$. Eight hours apart, mice received an equivalent volume of saline injection and were immediately confined in the other compartment for $30 \mathrm{~min}$ in the corresponding unit. These procedures were repeated for three consecutive days. On day 4 mice were placed in the center with guillotine doors open and started a 15 -min test. Durations (in second) for mice exploring in each of the three compartments were recorded automatically. Drug-induced CPP was represented by subtracting the time spent in saline-paired compartment from it in drug-paired compartment. With our experimental regimen, we found that 0.5 and $2.5 \mathrm{mg} / \mathrm{kg}$ were the minimal doses for methamphetamine and cocaine, respectively, to produce the conditioned place preferences. These minimal doses were doubled to produce robust drug-induced CPP in some experiments. A significant impairment in mouse locomotor activity and increased lethality was noticed following four doses of anisomycin at 100 , but not at $50 \mathrm{mg} / \mathrm{kg} / \mathrm{dose}$. Hence, a dose of $50 \mathrm{mg} / \mathrm{kg}$ anisomycin was used in this study. With the same criteria, cycloheximide at $15 \mathrm{mg} / \mathrm{kg} /$ dose was used.

\subsection{Western blotting analysis}

Western blotting was used to determine relative pCREB levels in various brain regions. Brain tissues (hippocampus, nucleus accumbens and prefrontal cortex including orbital cortex, prelimbic cortex, cingulated cortex and frontal association cortex) were immediately dissected at 10 and $30 \mathrm{~min}$ during and $60 \mathrm{~min}$ after the first psychotimulant-place pairing and homogenized in ice-cold phosphate buffered saline containing protease inhibitor cocktail (Roche, Basel, Switzerland). Homogenates were centrifuged at $15,000 \mathrm{~g}$ for $10 \mathrm{~min}$ at $4^{\circ} \mathrm{C}$, and the protein concentrations of supernatants were measured (Micro BCA protein assay kit, Pierce, Rockford, IL) and adjusted to an equivalent concentration. Supernatant samples were mixed with $4 \mathrm{X}$ sample buffer (Invitrogen, Carlsbad, CA), heated to $70{ }^{\circ} \mathrm{C}$ for $10 \mathrm{~min}$ in the presence of DTT, and separated on $4-12 \% \mathrm{Nu}$ PAGE gels (Invitrogen). The electrophoretically separated proteins were 
transferred to PVDF membranes (Bio-Rad, Hercules, CA), blocked with $5 \%$ non-fat milk in Tris buffered saline-Tween-20 $(20 \mathrm{mM}$ Tris- $\mathrm{HCl}, \mathrm{pH}$ 7.5, 0.5 M NaCl, 0.5\% Tween-20), probed with anti-phospho-Ser133CREB (pCREB) antibody (Upstate Biotechnology, Charlottesville, VA) in a 1:1000 dilution. The blots were developed with the Supersignal Chemiluminescent Substrates (Pierce Biotechnology, Rockford, IL) and the relative intensities of the bands were measured on a Biochemi imaging system (UVP, Upland, CA). The blots were then stripped with Restore Western Blot Stripping Buffer (Pierce Biotechnology) for $15 \mathrm{~min}$ and incubated with anti-pCREB or anti-CREB (Upstate Biotechnology) antibody in a 1:1000 dilution and appropriated secondary antibody. Control for protein loading was performed by reprobing membranes with a monoclonal anti$\beta$-actin antibody (1:5000, Chemicon, Temecula, CA).

\subsection{Stereotaxic infusion of CREB antisense oligonucleotides}

Stereotaxic guide cannula implantation (Coordinates: anteroposterior, $+1.3 \mathrm{~mm}$; lateral, $\pm 1.1 \mathrm{~mm}$; dorsoventral, $-3.3 \mathrm{~mm}$ ) was made under sodium pentobarbital anesthesia $(50 \mathrm{mg} / \mathrm{kg}$, i.p.). Bregma and the skull surface served as the stereotaxic zero point. The CREB antisense and scrambled sense oligodeoxynucleotides used in this study were obtained from MDBio Inc. (Taipei, Taiwan). The infusion cannula, a 30-gauge dental needle, was inserted into a 26-gauge guide cannula aimed at the nucleus accumbens. One microliter of oligodeoxynucleotide solution $(1 \mathrm{~nm} / \mu \mathrm{l})$ was infused bilaterally with a Hamilton $10-\mu \mathrm{l}$ microsyringe driven by a microdialysis pump (CMA/Microdialysis, Stockholm, Sweden) at a rate of $1 \mu 1 /$ min. After infusion, the injection cannula was left for an additional $4 \mathrm{~min}$ before withdrawal to reduce efflux of injection liquid along the injection tract. At the end of the test, the injection sites were confirmed immunohistochemistry by the infusion of biotinylated CREB antisense oligodeoxynucleotide and visualized using a Vectastain $\mathrm{ABC}$ kit (Vector Laboratories, Burlingame, CA).

\subsection{Statistics}

ANOVA was performed to determine group differences on druginduced conditioned place preference, followed by Bonferroni post hoc tests if appropriate. Mann-Whitney $U$ tests were employed to examine the group differences in the relative intensity of pCREB levels. Results were considered significant when $p$-values were $<0.05$, using a two-tailed test.

\section{Results}

\subsection{Effects of anisomycin and cycloheximide on memory formation of methamphetamine- and cocaine-induced conditioned place preferences}

Mice did not exhibit any place bias as saline was paired with both side compartments, indicating an unbiased design of our experimental protocol (Fig. 1A). Likewise, anisomycin $(50 \mathrm{mg} / \mathrm{kg}$, i.p.) treatment per se paired with both compartments did not cause place preference (Fig. 1A). Specifically, since anisomycin was given $30 \mathrm{~min}$ before the test drug, the effect of anisomycin pretreatment itself in place preference paradigm was examined. Thirty minutes after injections, anisomycin paired with one compartment and saline paired with the other compartment in the test chamber did not elicit any place preference (Fig. 1A), indicating no aversive effects caused by the test dose of anisomycin. We found that the minimal dose to establish reliable CPP for methamphetamine and cocaine was 0.5 and $2.5 \mathrm{mg} / \mathrm{kg}$, respectively. Anisomycin injection $30 \mathrm{~min}$ before cocaine-place pairings was found to abolish the cocaine-induced CPP $(p<0.01)$, whereas such anisomycin treatment did not alter the methamphetamine-induced CPP (Fig. 1A). Even more so, anisomycin injection $30 \mathrm{~min}$ before both drug and saline place pairings abolished the cocaine-induced CPP, whereas left the methamphetamineinduced CPP intact (Fig. 1A). In order to validate these findings, higher doses of methamphetamine and cocaine were used to produce more robust drug-induced CPP. Our results revealed that the anisomycin pretreatment still blocked the cocaine-induced CPP, even when $5 \mathrm{mg} / \mathrm{kg}$ of cocaine was used (Fig. 1B). However, methamphetamine $(1 \mathrm{mg} / \mathrm{kg})$-induced CPP remained unaffected by the anisomycin pretreatment (Fig. 1B). Treatment of another protein synthesis inhibitor, cycloheximide $(15 \mathrm{mg} / \mathrm{kg} /$ dose $)$, was used $2 \mathrm{~h}$ before the drug-place pairing (Lien et al., 2004). Mice did not exhibit any place bias as saline or cycloheximide was paired with both side compartments (Fig. 1C). Two hours after injections, cycloheximide paired with one compartment and saline paired with the other compartment did not elicit any place preference (Fig. 1A), indicating no aversive effects caused by the cycloheximide treatment protocol. We found that cycloheximide pretreatment mitigated the cocaine $(5 \mathrm{mg} / \mathrm{kg})$-induced CPP, while did not affect the methamphetamine $(1 \mathrm{mg} / \mathrm{kg})$-induced CPP (Fig. 1C). Considering that the time window allowed for anisomycin might not be appropriate for blocking the methamphetamine-induced CPP in previous experiments, we injected anisomycin $2 \mathrm{~h}$ before or after the drug-place pairings. Our results indicated that neither $2 \mathrm{~h}$ before nor $2 \mathrm{~h}$ after the drug-place pairing did anisomycin affect the methamphetamine-induced CPP (Fig. 1D). However, administration of anisomycin $2 \mathrm{~h}$ after, but not before the drug-place pairing, abolished the cocaine-induced CPP (Fig. 1D).

\subsection{Changes of $p C R E B$ levels in three brain regions during and after single session of drug-place pairing and the effects of CREB antisense oligodeoxynucleotide treatment}

In an attempt to examine the roles of pCREB in mediating the memory formation of psychostimulant-induced CPP, hippocampal, accumbal, and prefrontal cortical pCREB levels were analyzed 10, $30 \mathrm{~min}$ during and $60 \mathrm{~min}$ after the single drug-place pairing. Total CREB levels in these brain regions were not altered by the drug-place pairing experiences at any time point examined (data not shown). However, the levels of pCREB in the hippocampus were elevated in all three psychostimulant-place pairing conditions throughout the $90 \mathrm{~min}$ (Fig. 2A), indicating the relevance of hippocampal CREB activation to common stimuli in this paradigm. The pCREB levels in the prefrontal cortex also differed between the cocaine and methamphetamine groups in that methamphetamine animals exhibited an increase in pCREB during the 30-min drugplace pairing, whereas the cocaine group spared such an increase (Fig. 2C). Since consistent pCREB levels in the prefrontal cortex were found in the cocaine group, CREB 

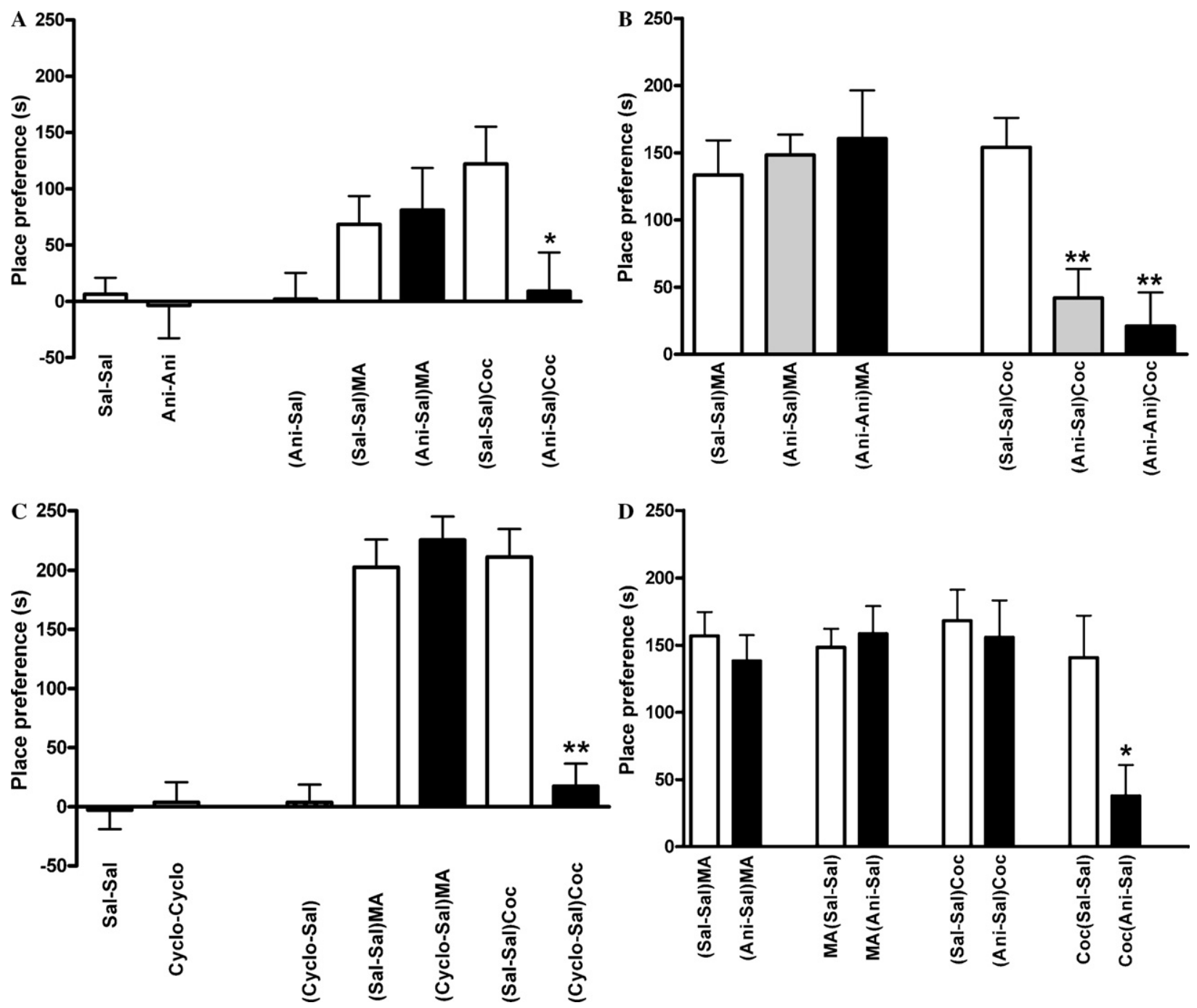

Fig. 1. Effects of anisomycin ( $50 \mathrm{mg} / \mathrm{kg} / \mathrm{dose})$ and cycloheximide $(15 \mathrm{mg} / \mathrm{kg} / \mathrm{dose})$ on cocaine and methamphetamine-induced conditioned place preference. (A) Saline (Sal-Sal) or anisomycin $(50 \mathrm{mg} / \mathrm{kg}$ ) (Ani-Ani) paired with two compartments did not elicit CPP. Thirty minutes after injection, anisomycin $(50 \mathrm{mg} / \mathrm{kg})$ paired with one compartment and saline paired with the other compartment [(Ani-Sal)] failed to elicit biased preference. With saline pretreatment $30 \mathrm{~min}$ before the pairings (in parentheses), methamphetamine [0.5 mg/kg, i.p.; (Sal-Sal)MA] and cocaine [2.5 mg/kg, i.p.; (Sal-Sal)Coc], demonstrated drug-induced CPP. Pretreatment of anisomycin $30 \mathrm{~min}$ before the drug-place pairings blocked the cocaine-induced CPP [(Ani-Sal) Coc], whereas such pretreatment did not alter the methamphetamine-induced CPP [(Ani-Sal)MA]. $n=8$ for each group. (B) Methamphetamine [1 mg/kg, i.p.; (Sal-Sal)MA] and cocaine [5 mg/kg, i.p.; (Sal-Sal)Coc] both demonstrated robust drug-induced CPP. Anisomycin pretreatment blocked the cocaineinduced CPP [(Ani-Sal)Coc and (Ani-Ani)Coc], whereas such pretreatments did not affect the methamphetamine-induced CPP [(Ani-Sal)MA or (AniAni)MA]. $n \geqslant 12$ for each group. (C) Saline (Sal-Sal) or cycloheximide (15 mg/kg) (Cyclo-Cyclo) paired with two compartments did not elicit CPP. Two hours after injection, cycloheximide $(15 \mathrm{mg} / \mathrm{kg})$ paired with one compartment and saline paired with the other place [(Cyclo-Sal)] did not elicit biased place preference. Methamphetamine [1.0 mg/kg, i.p.; (Sal-Sal)MA] and cocaine [5.0 mg/kg, i.p.; (Sal-Sal)Coc], demonstrated evident CPP. Pretreatment of cycloheximide $2 \mathrm{~h}$ before the drug-place pairings blocked the cocaine-induced CPP [(Cyclo-Sal)Coc], whereas such pretreatment did not alter the methamphetamine-induced CPP [(Cyclo-Sal)MA]. $n=8$ for each group. (D) Neither anisomycin injected $2 \mathrm{~h}$ before [(Ani-Sal)MA] nor $2 \mathrm{~h}$ after [MA(Ani-Sal)] the pairings affect the methamphetamine-induced CPP. Anisomycin injection $2 \mathrm{~h}$ after the pairings abolished the cocaine-induced CPP [Coc(Ani-Sal)]. $n=12$ for each group. ${ }^{*} p<0.05,{ }^{* *} p<0.01$.

activation in this region did not seem to play a critical role in the initial acquisition of cocaine-induced CPP.

In the nucleus accumbens, cocaine-place pairing produced an early and transient (only at $10 \mathrm{~min}$ ) elevation in pCREB levels, whereas methamphetamine-place pairings did not alter pCREB levels at any time point examined (Fig. 2B). These results imply that differential activation of CREB in the nucleus accumbens could be involved in drug-induced CPP between cocaine and methamphetamine.
To test this hypothesis, phosphorothioate-modified CREB antisense oligodeoxynucleotides (sequences: 5'-TGGT CATCTAGTCACCGGTG-3', $1 \mathrm{~nm} / 1 \mu \mathrm{l}$ ) (Guzowski \& McGaugh, 1997) were infused bilaterally into mouse nucleus accumbens 2-h before each drug-place pairing. At the end of the place preference test, injection sites were verified for each animal (Fig. 3A). Western blotting analysis indicated an approximately $50 \%$ decrease of accumbal CREB level $2 \mathrm{~h}$ after the CREB antisense oligodeoxynu- 


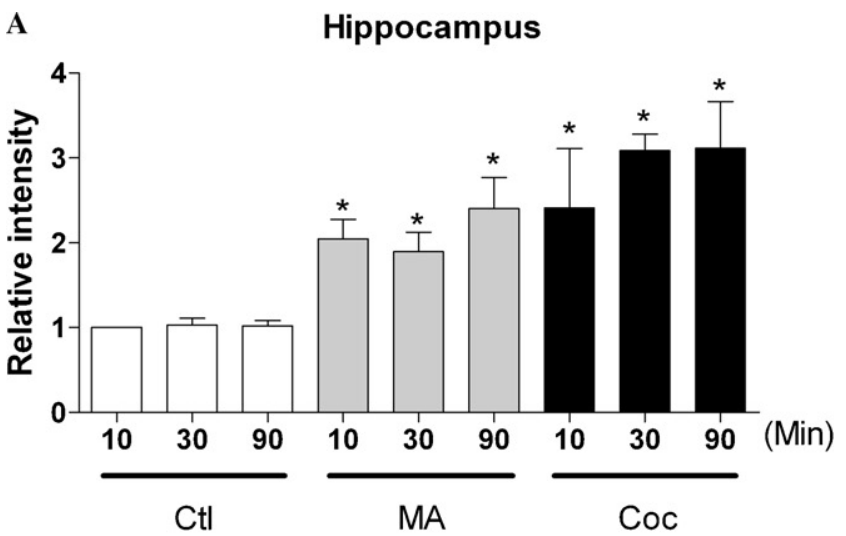

B

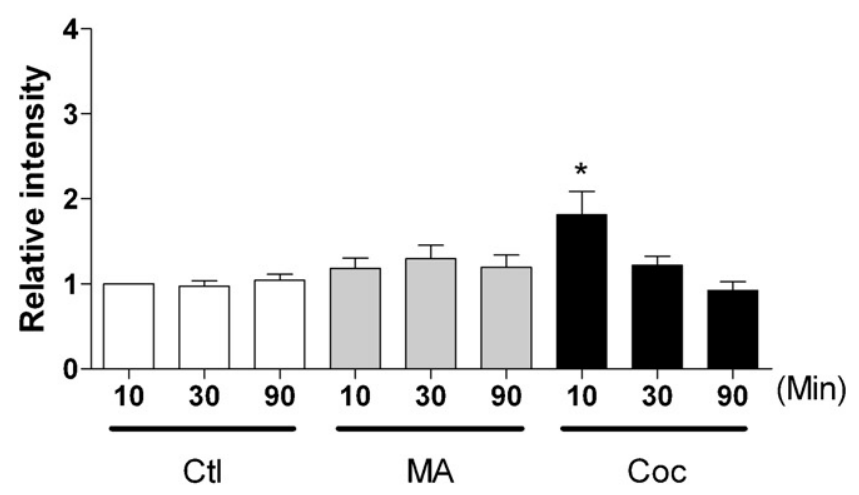

C

Prefrontal cortex

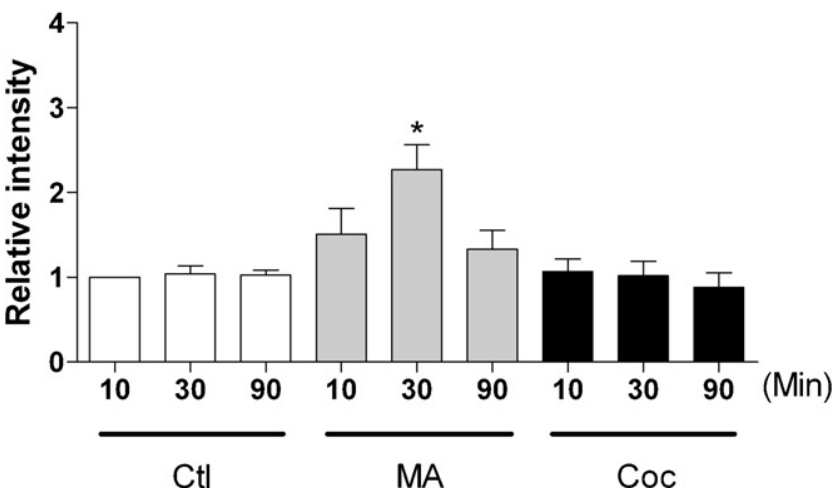

Fig. 2. Western immunoblotting of phosphorylated CREB (pCREB) levels in hippocampus, nucleus accumbens, and prefrontal cortex at 10 and 30 min during and $60 \mathrm{~min}$ after the first drug-place pairings. (A) Hippocampal pCREB levels were universally elevated following methamphetamine (MA; $1 \mathrm{mg} / \mathrm{kg}$ )- and cocaine (Coc; $5 \mathrm{mg} / \mathrm{kg}$ )-place pairings throughout the time points examined. (B) An early elevation $(10 \mathrm{~min})$ of accumbal pCREB levels was found only in Coc-treated group. (C) MAplace pairings significantly increased the prefrontal pCREB levels *Significantly higher than their respective controls, Mann-Whitney $U<0$, $p=0.05, n=3$ for each group.

cleotide infusion (Fig. 3B). CREB antisense- and scrambled sense-treated mice exhibited the same level of CPP for methamphetamine $(1 \mathrm{mg} / \mathrm{kg})$, whereas the CREB antisense treatment mitigated the cocaine $(5 \mathrm{mg} / \mathrm{kg})$-induced CPP (Fig. 3C). Moreover, the CREB antisense treatment had no effect on spontaneous locomotor activity (antisense:
$776.1 \pm 46.8$, scrambled sense: $796.2 \pm 58.8$; infrared breaking counts in mean \pm SEM for $3 \mathrm{~min}$ ).

\section{Discussion}

Our results showed that accumbal CREB phosphorylation and de novo protein synthesis was required for mediating the memory formation of the cocaine-induced conditioned place preference. These findings are in agreement with the notion that de novo protein synthesis plays a critical role in the long-term memory formation. Successful learning, memory stabilization and retrieval phases all are required for the expression of memory. We found that anisomycin treatment $30 \mathrm{~min}$ before and even $2 \mathrm{~h}$ after the learning phase of the cocaine-place pairings abolished the cocaine-induced place preference expression. However, anisomycin treatment $2 \mathrm{~h}$ before the cocaine-place pairings did not affect such memory formation. These data suggest that de novo protein synthesis at least play a critical role at the consolidation phase of the cocaine-induced CPP memory. Moreover, it is of importance to note that a quite long labile phase following the acquisition of cocaine-environmental cue association was observed. That is, the memory formation and stabilization process of the cocaine exposure-related experience might be susceptible to manipulation with a wide time window.

Although accumbal CREB phosphorylation and de novo protein synthesis seemed to play a critical role in mediating the memory formation of the cocaine-induced $\mathrm{CPP}$, whether $\mathrm{pCREB}$ and subsequent synthesized proteins are the memory substrates and/or whether the memory substrates are stored in the nucleus accumbens for the cocaine-induced place preference remain to be studied. Mice exhibited significant increases in accumbal pCREB levels at $10 \mathrm{~min}$ after the beginning of cocaine-place pairing. Such elevations returned to the basal levels rapidly even before the end of the cocaine-place pairing. Thus, an acute increase in accumbal pCREB levels is unlikely the memory substrate itself. Recently, a report addressed that medial prefrontal and amygdaloid gamma protein kinase $\mathrm{C}$ level could be used as an index for the retrieval of Pavlovian association memory trace in this drug-induced CPP paradigm (Thomas \& Everitt, 2001). Likewise, several lines of evidence did not seem to support that such memory substrate is stored in the nucleus accumbens. First, lesion of nucleus accumbens by intracranial 6-OHDA microinjection can not mitigate the established self-administration behavior for cocaine (Sizemore, Co, Koves, Martin, \& Smith, 2004). Second, chemical lesion of the afferent sources or efferent targets of nucleus accumbens can not abolish the established operant responses for obtaining cocaine (Pierce, Reeder, Hicks, Morgan, \& Kalivas, 1998; Robledo \& Koob, 1993; Smith et al., 2004). We, hereby, conclude that CREB activation in the nucleus accumbens and de novo protein synthesis mediate the formation of the cocaine-associated memory. However, their roles in retrieving the cocaineassociated memory deserve further studies. 
Although methamphetamine-induced conditioned place preference is characterized by its long-lasting property, our results demonstrated that methamphetamine-associated memory formation was independent of de novo protein synthesis. Similar phenomenon that certain long-term memories can emerge with over $90 \%$ absence of protein synthesis has been reported by others (Flood \& Cherkin, 1987; Judge \& Quartermain, 1982; Kameyama et al., 1986; Lattal \& Abel, 2001; Martinez et al., 1981; Quevedo et al., 1999; Rainbow et al., 1980). Nonetheless, our findings do not exclude the possibility that constitutively active pro-

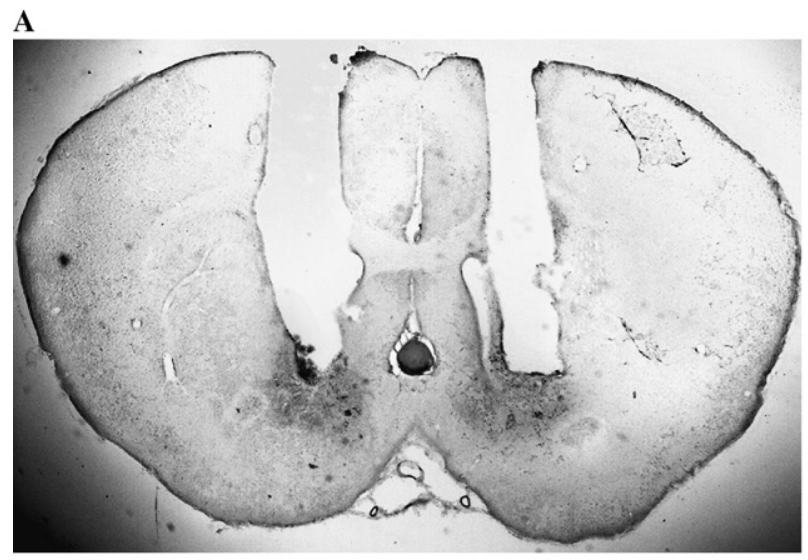

B
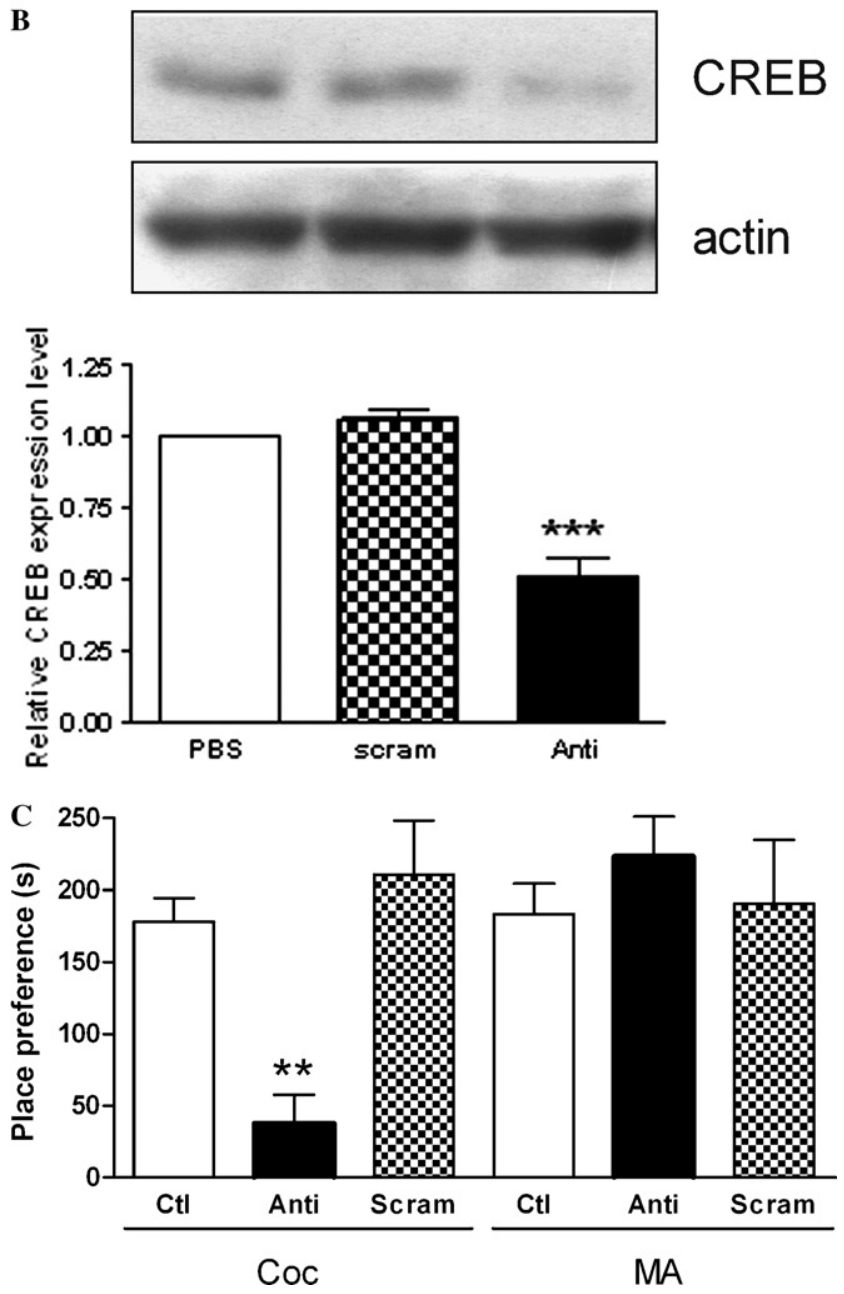

teins may participate in the memory formation for the methamphetamine-induced conditioned place preference. For example, post-translational modification of certain proteins nearby the synapses has been proposed as potential substrates for long-term memory (Routtenberg \& Rekart, 2005). A suite of protein modifications, including receptor modification and the subsequent receptor endocytosis/internalization (Nong, Huang, \& Salter, 2004), present in activated presynaptic and post-synaptic elements in response to the learning event can regulate the synaptic strength. Such changes in synaptic efficacy may, in turn, maintain the suite of protein post-translational modifications at the synapses. This positive feedback loop can spontaneously repeat and implicitly rehearse the synaptic efficacy changes to perpetuate the memory representations. With this proposal, new protein synthesis, at best, plays an optional role in replenishing the proteins available for the post-translational modifications.

Schnyer et al. (2004) reported that patients with medial prefrontal cortex lesion exhibited impaired recall, recognition and prospective feeling-of-knowing judgments. Perceptual hallucinations are common psychotic symptoms in methamphetamine addicts (Srisurapanont et al., 2003). We found that methamphetamine, but not cocaine, produced an increase in pCREB levels in the prefrontal cortex. It is likely that acute methamphetamine treatment at the doses that we used in this study produces hallucinogenic effects, whereas cocaine doses used do not cause these effects. Such methamphetamine-produced hallucinogenic effects may either interrupt the perceptual cue encoding or deteriorate the prospective memory monitoring, and consequently lead to the impairment of memory formation. Due to the possibility that methamphetamine-elicited hallucinogenic

Fig. 3. Effects of infusion of CREB antisense oligodeoxynucleotides into the nucleus accumbens on the cocaine- and methamphetamine-induced conditioned place preference. (A) An example of brain slice demonstrates the proper cannula placements and infusion sites. Immediately after the behavioral tests, mice were infused bilaterally by the injection cannulas with $1 \mu 1$ of biotinylated CREB antisense oligodeoxynucleotide. Two hours after injection, mice were anesthetized (Equithesin $5 \mathrm{ml} / \mathrm{kg}$, i.p.) and perfused transcardially with ice-cold saline and then $4 \%$ buffered paraformaldehyde. The brains were sectioned coronally at $40 \mu \mathrm{m}$ and stained by the Vectastain ABC kit (Vector Laboratories). The CREB antisense oligodeoxynucleotide positive stained areas (circles) confirms the successful injection of oligodeoxynucleotide into the nucleus accumbens. (B) Relative (to actin) CREB expression level after the injection of CREB antisense oligodeoxynucleotides. Western blotting analysis indicated that infusion of CREB antisense oligodeoxynucleotide (anti) to the nucleus accumbens reduced the expression of CREB to approximately $50 \%$ of that of PBS-infused group (PBS), while no such change was observed in the scramble oligodeoxynucleotide-infused (Scram) mice. ${ }^{* * *} p<0.001$; $n=5$ for each group. (C) CREB antisense oligodeoxynucleotides block the cocaine-induced place preference. Although cocaine $(5 \mathrm{mg} / \mathrm{kg})$ elicited a robust place preference (Ctl), bilateral infusion of CREB antisense oligodeoxynucleotides (anti) into the nucleus accumbens $2 \mathrm{~h}$ before the drug-place pairings blocked the cocaine (Coc)-induced place preference but did not affect the methamphetamine (MA)-induced place preference; ${ }^{* *} p<0.01 ; n=8$ for each group. CREB scrambled sense oligodeoxynucleotides (Scram) did not alter the drug-induced place preference. 
effects disrupt encoding of the environmental cues throughout the training sessions, the methamphetaminepaired compartment remains to be a novel place. Thus, the novelty-seeking instinct would drive the animals to spend more time to explore the methamphetamine-paired compartment than that of the familiar saline-paired compartment. Protein synthesis inhibitors used, in this regard, fail to reverse the methamphetamine-elicited hallucinogenic effects or interrupt the saline-compartment conditioning. Nonetheless, we do not negate the reinforcing and/or hedonic efficacy associated with methamphetamine. Numerous reports, after all, address that the operant behaviors can be supported by self-administration of both drugs and intraaccumbens amphetamine injection supported conditioned place preference (Josselyn \& Beninger, 1993; Vezina, 2004). It is of importance to note that cocaine can achieve more complete and faster self-administration levels following the autoshaping procedure compared to amphetamine as these two drugs were used at equally potent doses (Carroll \& Lac, 1997). Another explanation for the differential roles of de novo protein synthesis in the methamphetamine- and cocaine-induced conditioned place preference lies in the distinctive pharmacological and biochemical profiles of these two drugs. Cocaine, a dopamine reuptake blocker, binds directly to dopamine transporter (DAT) and inhibits the activity of DAT. Cocaine has been reported to cause a rapid increase in dopamine uptake and plasmalemmal DAT expression in synaptosome preparation (Daws et al., 2002; Fleckenstein et al., 1999). In contrast, methamphetamine, a frequently classified dopamine releaser, redistributed vesicular dopamine into cytosol and stimulated reverse transport and/or channel-like activity of DAT (Kahlig et al., 2005; Sulzer et al., 1995). Methamphetamine has been found to rapidly decrease dopamine uptake and bi-physically regulate membrane DAT expression in synaptosome preparation (Fleckenstein, Metzger, Gibb, \& Hanson, 1997; Johnson, Furman, Zhang, Guptaroy, \& Gnegy, 2005). Moreover, cocaine-produced extracellular dopamine increase rendered vesicular monoamine transporter-2 (VMAT-2) trafficking from axolemma to cytoplasm (Brown, Hanson, \& Fleckenstein, 2001), whereas methamphetamine resulted in VMAT-2 trafficking from cytoplasm to an unidentified subcellular region (Riddle, Topham, Haycock, Hanson, \& Fleckenstein, 2002). Thus, although methamphetamine and cocaine both possess the reinforcing/hedonic efficacy to support the Pavlovian conditioning responses, the differential pharmacological and biochemical characteristics between methamphetamine and cocaine suggest that various molecular pathways could be involved in the reinforcing efficacy-dependent memory associated with these two drugs. In conclusion, we suggest that methamphetamine-associated memory formation is independent of de novo protein synthesis. Formation of the cocaine-associated memory, in part, relies on the accumbal CREB activation and the newly synthesized proteins, especially at the memory consolidation phase.

\section{Acknowledgments}

This research is supported by ROC National Science Council grants $922413 \mathrm{H} 006008,922321 \mathrm{~B} 006004$, and the Ta-You Wu Memorial Award to L.Y.

\section{References}

Achat-Mendes, C., Anderson, K. L., \& Itzhak, Y. (2003). Methylphenidate and MDMA adolescent exposure in mice: long-lasting consequences on cocaine-induced reward and psychomotor stimulation in adulthood. Neuropharmacology, 45, 106-115.

Bonci, A., Bernardi, G., Grillner, P., \& Mercuri, N. B. (2003). The dopamine-containing neuron: maestro or simple musician in the orchestra of addiction? Trends in Pharmacological Sciences, 24, 172-177.

Brown, J. M., Hanson, G. R., \& Fleckenstein, A. E. (2001). Cocaineinduced increases in vesicular dopamine uptake: role of dopamine receptors. The Journal of Pharmacology and Experimental Therapeutics, 298, 1150-1153.

Cannon, L. M., \& Bseikri, M. R. (2004). Is dopamine required for natural reward? Physiology \& behavior, 81, 741-748.

Carroll, M. E., \& Lac, S. T. (1997). Acquisition of i.v. amphetamine and cocaine self-administration in rats as a function of dose. Psychopharmacology, 129, 206-214.

Daws, L. C., Callaghan, P. D., Moron, J. A., Kahlig, K. M., Shippenberg, T. S., Javitch, J. A., \& Galli, A. (2002). Cocaine increases dopamine uptake and cell surface expression of dopamine transporters. Biochemical and Biophysical Research Communication, 290, 1545-1550.

Ezzeddine, Y., \& Glanzman, D. L. (2003). Prolonged habituation of the gill-withdrawal reflex in Aplysia depends on protein synthesis, protein phosphatase activity, and postsynaptic glutamate receptors. Journal of Neuroscience, 23, 9585-9594.

Fleckenstein, A. E., Haughey, H. M., Metzger, R. R., Kokoshka, J. M., Riddle, E. L., Hanson, J. E., et al. (1999). Differential effects of psychostimulants and related agents on dopaminergic and serotonergic transporter function. European Journal of Pharmacology, 382, 45-49.

Fleckenstein, A. E., Metzger, R. R., Gibb, J. W., \& Hanson, G. R. (1997). A rapid reversible change in dopamine transporters induced by methamphetamine. European Journal of Pharmacology, 323, R9-R10.

Flood, J. F., \& Cherkin, A. (1987). Fluoxetine enhances memory processing in mice. Psychopharmacology (Berlin), 93, 36-43.

Frank, D. A., \& Greenberg, M. E. (1994). CREB: a mediator of long-term memory from mollusks to mammals. Cell, 79, 5-8.

Goelet, P., Castellucci, V. F., Schacher, S., \& Kandel, E. R. (1986). The long and the short of long-term memory - a molecular framework. Nature, $322,419-422$

Guzowski, J. F., \& McGaugh, J. L. (1997). Antisense oligodeoxynucleotide-mediated disruption of hippocampal cAMP response element binding protein levels impairs consolidation of memory for water maze training. Proceedings of the National Academy of Sciences of the United States of America, 94, 2693-2698.

Johnson, L. A., Furman, C. A., Zhang, M., Guptaroy, B., \& Gnegy, M. E. (2005). Rapid delivery of the dopamine transporter to the plasmalemmal membrane upon amphetamine stimulation. Neuropharmacology, $49,750-758$.

Josselyn, S. A., \& Beninger, R. J. (1993). Neuropeptide Y: intraaccumbens injections produce a place preference that is blocked by cisflupenthixol. Pharmacology Biochemistry \& Behavior, 46, 543-552.

Judge, M. E., \& Quartermain, D. (1982). Alleviation of anisomycininduced amnesia by pre-test treatment with lysine-vasopressin. Pharmacology, Biochemistry \& Behavior, 16, 463-466.

Kahlig, K. M., Binda, F., Khoshbouei, H., Blakely, R. D., McMahon, D. G., Javitch, J. A., et al. (2005). Amphetamine induces dopamine efflux through a dopamine transporter channel. Proceedings of the National Academy of Sciences of the United States of America, 102, 3495-3500 
Kameyama, T., Nabeshima, T., \& Kozawa, T. (1986). The antagonistic effects of naloxone on cycloheximide and anisomycin-induced amnesia. Pharmacology Biochemistry \& Behavior, 25, 567-572.

Kandel, E. R. (2001). The molecular biology of memory storage: a dialogue between genes and synapses. Science, 294, 1030-1038.

Lattal, K. M., \& Abel, T. (2001). Different requirements for protein synthesis in acquisition and extinction of spatial preferences and contextevoked fear. Journal of Neuroscience, 21, 5773-5780.

Lien, W.-H., Yeh, T.-L., Yang, Y.-K., Cherng, C. G., Chen, H.-H., Chen, P. S., et al. (2004). Cycloheximide enhances maintenance of methamphetamine-induced conditioned place preference. Chinese Journal of Physiology, 47, 23-30.

Martinez, J. L., Jr., Jensen, R. A., \& McGaugh, J. L. (1981). Attenuation of experimentally-induced amnesia. Progress in Neurobiology, 16, 155186.

McClung, C. A., \& Nestler, E. J. (2003). Regulation of gene expression and cocaine reward by CREB and DeltaFosB. Nature Neuroscience, 6, 1208-1215.

Nong, Y., Huang, Y-Q., \& Salter, M. W. (2004). NMDA receptors are movin' in. Current Opinion in Neurobiology, 14, 353-361.

Pierce, R. C., Reeder, D. C., Hicks, J., Morgan, Z. R., \& Kalivas, P. W. (1998). Ibotenic acid lesions of the dorsal prefrontal cortex disrupt the expression of behavioral sensitization to cocaine. Neuroscience, 82, 1103-1114

Quevedo, J., Vianna, M. R., Roesler, R., de-Paris, F., Izquierdo, I., \& Rose, S. P. (1999). Two time windows of anisomycin-induced amnesia for inhibitory avoidance training in rats: protection from amnesia by pretraining but not pre-exposure to the task apparatus. Learning Memery, 6, 600-607.

Rainbow, T. C., Hoffman, P. L., \& Flexner, L. B. (1980). Studies of memory: a reevaluation in mice of the effects of inhibitors on the rate of synthesis of cerebral proteins as related to amnesia. Pharmacology Biochemistry \& Behavior, 12, 79-84.

Ramirez, R. R., Gandhi, C. C., Muzzio, I. A., \& Matzel, L. D. (1998). Protein synthesis-dependent memory and neuronal enhancement in Hermissenda are contingent on parameters of training and retention. Learning Memory, 4, 462-477.

Riddle, E. L., Topham, M. K., Haycock, J. W., Hanson, G. R., \& Fleckenstein, A. E. (2002). Differential trafficking of the vesicular monoamine transporter-2 by methamphetamine and cocaine. European Journal of Pharmacology, 449, 71-74.

Robledo, P., \& Koob, G. F. (1993). Two discrete nucleus accumbens projection areas differentially mediate cocaine self-administration in the rat. Behavioural Brain Research, 55, 159-166.

Routtenberg, A., \& Rekart, J. L. (2005). Post-translational modification as the substrate for the long-lasting memory. Trends in Neurosciences, 28, 12-19.

Schnyer, D. M., Verfaellie, M., Alexander, M. P., LaFleche, G., Nicholls, L., \& Kaszniak, A. W. (2004). A role for right medial prefrontal cortex in accurate feeling-of-knowing judgments: evidence from patients with lesions to frontal cortex. Neuropsychologia, 42, 957-966.

Sizemore, G. M., Co, C., Koves, T. R., Martin, T. J., \& Smith, J. E. (2004). Time-dependent recovery from the effects of 6-hydroxydopamine lesions of the rat nucleus accumbens on cocaine self-administration and the levels of dopamine in microdialysis. Psychopharmacology, 171, 413-420

Smith, J. E., Co, C., Yin, X., Sizemore, G. M., Liguori, A., Johnson, W. E., 3rd, et al. (2004). Involvement of cholinergic neuronal systems in intravenous cocaine self-administration. Neuroscience \& Biobehavioral Reviews, 27, 841-850.

Srisurapanont, M., Ali, R., Marsden, J., Sunga, A., Wada, K., \& Monteiro, M. (2003). Psychotic symptoms in methamphetamine psychotic inpatients. International Journal of Neuropsychopharmacology, 6, 347352.

Stiedl, O., Palve, M., Radulovic, J., Birkenfeld, K., \& Spiess, J. (1999). Differential impairment of auditory and contextual fear conditioning by protein synthesis inhibition in C57BL/6N mice. Behavioral Neuroscience, 113, 496-506.

Sulzer, D., Chen, T., Lau, Y. Y., Kristensen, H., Rayport, S., \& Ewing, A. (1995). Amphetamine redistributes dopamine from synaptic vesicles to the cytosol and promotes reverse transport. The Journal of Neuroscience, $15,4102-4108$.

Thomas, K. L., \& Everitt, B. J. (2001). Limbic-cortical-ventral striatal activation during retrieval of a discrete cocaine-associated stimulus: a cellular imaging study with gamma protein kinase c expression. The Journal of Neuroscience, 21, 2526-2535.

Vezina, P. (2004). Sensitization of midbrain dopamine neuron reactivity and the self-administration of psychomotor stimulant drugs. Neuroscience \& Biobehavioral Reviews, 27, 827-839. 